\title{
REPENSAR LA EDUCACIÓN Y LA PEDAGOGÍA: ALGUNAS REFLEXIONES CRÍTICAS
}

\author{
Lode E. Cascante Gómez ${ }^{1}$ \\ Académica e investigadora del CIDE-UNA \\ Heredia, Costa Rica \\ Francisco González Alvarado ${ }^{2}$ \\ Académico e investigadora del CIDE-UNA \\ Heredia, Costa Rica
}

\begin{abstract}
"Aunque quizá estemos acostumbrados a pensar en la práctica como una mera actividad, puede demostrarse que el sentido y la significación de la práctica educativa y pedagógica se construye en los planos social, histórico y político, y que sólo puede entenderse de forma interpretativa y crítica".
\end{abstract}

Wilfred Carr, 1999

Recibido: 10 de noviembre, 2007 - Aprobado: 20 de marzo, 2008

Resumen: Reflexionar sobre la escuela como un lugar de construcción cultural permanente, influida por múltiples relaciones; pero, a la vez, como el lugar donde éstas se mediatizan y se construyen otras nuevas, se torna necesario. Valorar que los diseños curriculares explicitan y condicionan las prácticas escolares, y determinan, en buena medida, la intencionalidad de las prácticas pedagógicas es una cuestión que no se debe dejar de lado, en cualquier análisis sobre la educación y la pedagogía.

Estas ideas constituyen los ejes sobre los cuales se articulan las reflexiones de este escrito, las cuales, lejos de plantearse, sólo como cuestiones, puramente, teóricas, se visualizan como posibilidades prácticas. Los argumentos que aquí se expresan recogen las preocupaciones, más importantes, de los autores, y constituyen el resultado aproximado de la lectura y la reflexión sobre la práctica pedagógica personal, desde las aulas universitarias y la experiencia de trabajar con jóvenes de secundaria.

Palabras clave: Educación, pedagogía, interculturalidad, flexibilidad curricular, cultura experencial.

Abstract: Certainly it becomes necessary to reflect on the school not only as a place of permanent cultural construction, influenced by multiple relationships, but also on how it becomes the place where these connections band together and set up new ones.

Master en Pedagogía de la Diversidad y Licenciada en la Enseñanza de los Estudios Sociales por la Universidad Nacional de Costa Rica. Coautora de libros de texto para la enseñanza de los Estudios Sociales y artículos relacionados con la educación y la pedagogía. Coordinadora académica de primaria en el Colegio Marista. Académica e investigadora de la UNA desde 1995. lodelena@yahoo.com

2. Máster en Educación, Licenciado en la Enseñanza de los Estudios Sociales y Bachiller en Historia por la Universidad Nacional de Costa Rica. Coautor de libros de texto para la enseñanza de los Estudios Sociales y artículos relacionados con la educación y la pedagogía. Subdirector de la División de Educología de la Universidad Nacional. Académico e Investigador de la UNA desde 1995. frajoga@yahoo.com 
In every analysis on education and pedagogies, it must not be left behind the fact that curricular designs explicit and condition the school practices. Likewise, these outlines also determine the intentionality of the teaching practices.

These ideas constitute the basis guiding the reflections of this investigation, which more than appearing as theoretical issues, are actually visualized like practical possibilities. The arguments that are expressed here gather the most significant concerns conveyed by the authors. They account for the approximate outcome of the reading and the reflection on the personal teaching practice gained within the university classrooms and the experience of working with high school students.

Key words: Education, pedagogies, inter-culture, curricular flexibility, experiential culture.

\section{El punto de partida}

Reflexionar sobre la educación y la pedagogía no es un tema nuevo y, posiblemente, nunca lo será; sin embargo, ello no supone que su abordaje deba relegarse al cajón de los recuerdos o que tenga que limitarse su discusión al mero recuento de estadísticas o a la presentación de técnicas didácticas carentes de contextualización. Por el contrario, el debate debe partir de la mirada crítica al interior de los escenarios educativos y su intención fundamental, no por ello la única, deberá orientarse a desmenuzar la interdependencia del sistema educativo con el resto del entramado social y cultural que le da vida y orientación.

Por ello, las siguientes páginas tienen la intención de reflexionar desde una mirada crítica, en tres aspectos de la educación y la pedagogía que, a nuestro juicio, constituyen ejes sobre los cuales se debe orientar la discusión en estos campos, especialmente porque envuelven esa dinámica interdependiente entre sociedad, en el amplio sentido del concepto y la educación. Éstos giran sobre los siguientes: las exigencias y las resistencias históricas inmediatas, que delimitan el accionar de los sistemas educativos hoy en día; la necesidad de valorar con ojos críticos las continuidades y los cambios de la cultura escolar y, por último, la interpelación sobre las implicaciones que tiene, en los modelos de formación de docentes, la separación de la teoría y la práctica pedagógica. La discusión de los aspectos señalados se aborda en apartados independientes, con el objetivo de llamar la atención del lector sobre tres puntos específicos, lo 
que no necesariamente implica, el fraccionamiento incoherente de la temática; por el contrario: se intenta abordar un mismo objeto de estudio desde plataformas complementarias.

Tampoco se pretende, al escribir estas líneas, dar por agotada la discusión sobre un tema que, como ya se mencionó, es posible que nunca se acabe, sino que, más bien, se trata de continuar con la discusión aportando puntos de vista que recojan algunas de las preocupaciones más apremiantes de quienes escriben y constituyen el resultado aproximado de la lectura y la reflexión sobre las prácticas pedagógicas personales, que se han ido construyendo desde las aulas universitarias y en la interacción con jóvenes de secundaria.

\section{Posmodernidad y educación: exigencias y resistencias}

Con el final de las economías de planificación central, especialmente las de Europa Oriental, proceso que para muchos historiadores alcanza su cúspide con las denominadas revoluciones de 1989 en los países del bloque soviético o democracias socialistas, unido a la incorporación de la era de la electrónica y la digitalización a los escenarios de la producción de mercancías, su circulación y consumo, con mayor cobertura a finales de la década de los setenta en los países del capitalismo avanzado, y una década más en aquellos periféricos, las relaciones sociales, políticas y económicas entre los ciudadanos del planeta, adquieren nuevos significados y se despliegan nuevas visiones sobre la geopolítica, la cultura y, por supuesto, la educación. Para muchos, se llega al fin de la modernidad y de sus ideales inspirados en las revoluciones burguesas del siglo XVII, y se inicia un nuevo orden mundial, una era "posmoderna".

Nuevos conceptos dominan los lenguajes productivos, se incorporan las tecnologías de la información y la comunicación (TICS) en la vida cotidiana de un buen número de personas y la integración económica, y los tratados de libre comercio dibujan un nuevo orden planetario. Las economías flexibles, con sus modernas definiciones y distribuciones de tareas, exigen nuevas cualificaciones y destrezas a los trabajadores y a quienes los forman.

De ahí que las tareas encomendadas a los sistemas educativos adquieran, también, nuevas exigencias, especialmente, a la luz de las nacientes formas de organización de la 
producción globalizante, caracterizadas, entre otros aspectos, por imponer una serie de técnicas de trabajo y procesos laborales más flexibles, articuladas, de forma directa, a los intereses del capital transnacional; “mientras la estandarización, la demarcación de tareas y la producción en masa caracterizaban las economías modernas, las posmodernas, rompen las demarcaciones de los trabajos tradicionales e introducen intersecciones y rotaciones en las asignaciones de tareas y en la descripción de las mismas. También facilitan los ajustes rápidos y fáciles en el volumen de la fuerza de trabajo, mediante la jornada parcial y el trabajo temporal, el despido, etcétera" (Hargreves, 2003, p. 25).

Orientados, entre otros aspectos, por exigencias como las señaladas, los sistemas educativos difundidos por los estados, insisten en adoptar, de manera vertical y con criterios tecnocráticos, concepciones y formas de organización de tipo empresarial, en los que sobresalen nociones como polifuncionalidad, competencias, desarrollo de capacidades cognoscitivas, cambio dentro del cambio, flexibilidad, adaptación, diversidad, tecnología, entre otros. Nociones que son incorporadas en los diseños curriculares y en las prácticas pedagógicas, sin reflexión, o con muy poca.

Se exige de los docentes el desarrollo de espacios de aprendizaje con características como las descritas, muchos de ellos las aceptan sin conocer sus verdaderos fundamentos, y aquellos que opongan resistencia son considerados como profesores tradicionales, con prácticas pedagógicas de la modernidad. En este sentido, es preponderante reflexionar, de manera crítica, sobre la visión estrictamente técnico-instrumental que promueven algunos modelos teóricos en la educación, los cuales enfatizan, estrictamente, sobre los mecanismos psicológicos que intervienen en los aprendizajes, y dejan de lado cuestiones prioritarias, tales como su sentido político-cultural y su posibilidad significativa crítica.

Puede que se corra un riesgo si se adopta de forma ingenua esta tendencia, de construir diseños curriculares y desarrollar prácticas pedagógicas, que sustituyan las aspiraciones modernas de autonomía y emancipación, por racionalidades posmodernas tecnocráticas, en las que interesa más la formación de competencias, exclusivamente, destinadas a fortalecer y a preservar el nuevo mundo del trabajo, por encima de la educabilidad humana en el más amplio sentido de la palabra, resistencia importante de fortalecer ante los embates de una educación 
excluyente y que, de manera continua, promueve la adopción de paradigmas centrados en la conservación del estado actual de las cosas.

Negar la relevancia de flexibilizar los currículos educativos no tiene ni objeto, ni futuro; sin embargo, hacerlo ingenuamente y sin visualizar su impacto en el marco de una sociedad que privilegia grandes asimetrías, mucho menos. Al respecto, señala Menzies (1989, citado por Hargreves, 2003): "la flexibilidad puede crear ambientes de trabajo y estructuras sociales elitistas y segregadoras, reservando la autonomía, el criterio propio y el trabajo más significativo para las pequeñas elites técnicas, mientras que la mano de obra restante queda relegada a los trabajos de bajo nivel, de media jornada, temporales, sin seguridad social y asignados de forma errática" (p. 77).

Algunas de las observaciones señaladas, por desgracia, comienzan a visualizarse con cierta recurrencia entre los docentes que se desempeñan en nuevos escenarios laborales, los cuales se ven obligados a desempeñar más de una jornada laboral, en muchos de los casos, en espacios informales de la economía, realizando tareas de transportistas al margen de la legalidad, vendedores de artículos de uso personal, o improvisando salones de clase en sus mismas viviendas. $\mathrm{Y}$, en el mejor de los casos, compartiendo unas pocas lecciones con otros centros educativos, lo que, de seguro, repercute y no, necesariamente, de forma positiva, en su calidad de vida y, por supuesto, en el desempeño de su labor profesional.

Por más que insistiéramos en negarlo, asistimos a un cambio de época que ha trastrocado todos los niveles de la vida social, cultural y económica. El fin de los estados modernos, consolidados con la llustración y la Modernidad y caracterizados por una estructura cimentada en los conceptos de nación, gobierno, población y territorio, dan paso, poco a poco, a otros, que se fundamentan en categorías de supranacionalidad o supraestados; en estos últimos, lo local, ha cedido terreno ante lo global, lo universal. De esta forma, la geografía política planetaria adquiere dimensiones nuevas, y las corporaciones financieras han comenzado a expandir sus intereses por encima de las fronteras nacionales, empleando, para ello, los recursos locales, mano de obra, infraestructura y echando mano de sistemas educativos muy poco críticos y contestatarios, a pesar de que, en todas las reformas educativas, especialmente 
en el ámbito latinoamericano, se profesan paradigmas centrados en la desarrollo social; ¿crecimiento económico?.

Entre las principales preocupaciones, desde una postura reflexiva, que aquejan a los sistemas educativos en la actualidad, a la luz de la contextualización arriba descrita, sobresalen: la revisión crítica de la cultura escolar y sus formas de explicar, comunicar y valorar el conocimiento y los aprendizajes especialmente locales, y la necesidad de ofrecer procesos de reflexión profunda sobre la obligación de acercar la teorización sobre la educación, con prácticas pedagógicas concretas, en el marco de modelos de formación de docentes, pertinentes, flexibles y con sentido cultural. Estas preocupaciones serán el objeto de la reflexión en los siguientes apartados.

\section{Cultura escolar y prácticas pedagógicas: continuidades y rupturas}

Cincuenta años después de iniciada la llamada revolución cognoscitiva del aprendizaje, que, según el psicólogo educativo norteamericano, Jerome Bruner, arrancó cuando en el Congreso de Psicología de Viena, en 1953, los exponentes soviéticos iniciaron sus intervenciones sin hacer una genuflexión a Pavlov, es, en realidad, pobre el impacto que ha tenido ésta, en la modificación de las formas, concepciones y prácticas pedagógicas, desarrolladas, cotidianamente, al interior de los centros de estudio, desde los diferentes ámbitos del quehacer educativo formal: escolar, colegial, universitario, sin olvidar los modelos no formales.

Sin embargo, la investigación en la educación y, concretamente, en la psicología del aprendizaje, ha sido exhaustiva en estos años, y ha permitido aportar información relevante en cuanto a los procesos cognoscitivos y socioculturales involucrados con el aprendizaje, en particular, y con las prácticas pedagógicas, en general. Prueba de ello, lo constituyen las aportaciones derivadas de la neurociencia en la comprensión de los fenómenos biológicos, que tienen lugar en el cerebro humano. Los aportes de la antropología y la sociología educativa, en la comprensión de los escenarios educativos como espacios culturales dinámicos, unidos a los 
adelantos de la era de la digitalización de la vida social y cultural, han facilitado la incorporación de recursos tecnológicos para la enseñanza, y han abierto la puerta para la entrada de la informática como herramienta para desarrollar espacios de aprendizaje más dinámicos y flexibles.

A pesar de que, en el plano teórico y experimental, los recursos bibliográficos, materiales didácticos y fuentes de información con que cuentan los educadores, son numerosos, atractivos $y$, potencialmente, liberadores de aprendizaje, la inmensa mayoría de los centros de formación universitaria y por consiguiente, los docentes y los mismos estudiantes, continúan arrastrando el pesado lastre de la tradición asociacionista del aprendizaje, por lo que los escenarios educativos continúan siendo espacios ritualísticos que se alejan de la realidad científica, tecnológica y de la vida misma. El principio de teoría de la copia, que sostiene que aprender es reproducir la realidad y el principio de equipotencialidad, según el cual, los procesos de aprendizaje son universales, son los mismos en todas las tareas, en todas las personas e, incluso en todas las especies; constituyen los pilares fundamentales de esta tradición educativa.

Por esto, resulta fácil encontrar en los espacios dedicados a la educación, experiencias pedagógicas distinguidas por transmitir, a manera de copia, contenidos que son vaciados en los cerebros de quienes aprenden, sin tratamiento idiosincrático ni experiencial alguno, concepciones sobre el mundo, la ciencia y la historia, en muchos casos, obsoletas, confusas o erróneas. Propuestas curriculares homogenizantes, diseñadas, de forma técnica, desde oficinas administrativas, pero pobremente alimentadas por ideas de quienes las van a poner en marcha, cuando bajan a las aulas, los docentes sustituyen los antiguos planeamientos por otros enriquecidos de manera técnica; pero en la práctica concreta, no pasa nada. Posiciones autoritarias ocultas bajo el velo del activismo pedagógico, el cual transmite entusiasmo y entretiene, pero aporta, muy poco, a la tarea de desarrollar procesos mentales superiores de pensamiento y de generar estrategias de transformación social, esto es: vino viejo en botellas nuevas.

Sin embargo, creer que el problema del aprendizaje se reduce, exclusivamente, al dominio, en las prácticas pedagógicas, de unos principios teóricos del aprendizaje, o que la responsabilidad es de los agentes directos de ese proceso: docentes y estudiantes, es sencillo. 
No obstante, el problema es más complejo, en especial, en el ámbito de la enseñanza media. Y es que los escenarios de aprendizaje e instrucción, muchas veces, no están pensados teniendo en cuenta las características de los estudiantes y de sus docentes, su cultura experiencial, ni tampoco cuentan con los recursos infraestructurales y didácticos apropiados o mínimos y, que el Estado, por lo menos en Latinoamérica, redefine, de manera excluyente, su proyecto educativo, dejando por fuera, en el amplio sentido de la palabra, los grupos sociales menos favorecidos.

El consenso emergente entre los diversos sectores sociales, en cuanto a las razones explicativas de la crisis de la concepción tradicional del aprendizaje, es que se debe, no tanto al empuje de la investigación científica y de las nuevas teorías psicológicas, sino a la conjunción de diversos cambios sociales, tecnológicos y culturales. A partir de ellos, esa imagen tradicional experimenta un deterioro progresivo, a causa del desajuste creciente entre lo que la sociedad pretende que sus ciudadanos aprendan y los procesos que ésta pone en marcha para lograrlo. Las voluntades políticas continúan siendo limitadas.

Es urgente que paralelo al mejoramiento cuantitativo y cualitativo de las condiciones materiales para brindar una educación de acceso universal, se faciliten los espacios adecuados para un mejor conocimiento del accionar del aprendizaje como proceso psicológico y cultural. En este sentido, es inminente la necesidad de acercar la sociedad costarricense, en particular, y latinoamericana, en general, a lo que algunos investigadores educativos renombrados, entre ellos Pozo (2001) denominan, la nueva cultura del aprendizaje, distinguida, entre otros aspectos, por una educación generalizada y por una formación permanente y masiva, por una saturación informativa producida por los nuevos sistemas de producción, comunicación y conservación de la información, y por un conocimiento descentralizado y diversificado. Según Pozo, esta sociedad del aprendizaje continuado, de la explosión informativa y del conocimiento relativo genera unas demandas de aprendizaje que no pueden compararse con las de otras épocas pasadas, tanto en calidad como en cantidad.

Sin nuevas estrategias, articuladas a políticas estatales, que propendan el cambio de timón, en cuanto al enfoque sobre el aprendizaje y sus consecuentes prácticas pedagógicas, las demandas sociales desbordarán, con creces, las capacidades y recursos de la mayor parte de los 
sujetos de aprendizaje, produciendo un efecto paradójico de deterioro del aprendizaje mismo. Mientras el Estado no asuma, con responsabilidad, la tarea que, constitucionalmente, le corresponde, en cuanto al papel de la educación como medio de desarrollo, y las instituciones y organizaciones educativas, Ilámese, universitarias públicas o privadas, magisteriales, gremiales o estudiantiles, no profundicen en la revisión de sus estrategias de aprendizaje, y logren formular propuestas educativas alternativas a la tan criticada enseñanza tradicional, es poco el cambio que se puede captar.

Por ello, para muchos educadores, la escuela, en el amplio sentido de la expresión, se ha convertido en una carga cada vez más pesada, sobre la cual, con mayor frecuencia, se añaden nuevas tareas y cuya legitimidad se ve debilitada. Las razones de tal suceso son variadas y abundantes, al igual que sus consecuencias; sin embargo, en un aspecto casi todos los educadores están convencidos: la escuela, como espacio cultural, ha dejado de ser lo que fue en los últimos treinta años, y será otra cosa muy distinta en los próximos diez. Pero esto no significa que tenemos nuevas escuelas, sino que las formas tradicionales de control y las consecuentes manifestaciones de resistencia han variado, al punto de ruptura entre unas y otras, de ahí que, para no pocos docentes, especialmente los que se desempeñan en ambientes de enseñanza primaria y media, el oficio apasionado de educar, se haya transformado en una tarea difícil de sobrellevar.

Las transformaciones políticas, económicas y sociales, en el marco de unas relaciones, cada vez más globales, imponen a la educación un conjunto de cambios que interpelan nuevas formas de comprender la escuela. La visión romántica, articulada a la consideración de los espacios educativos como lugares estables y permanentes, ya no existe más, a pesar del esfuerzo que realizan algunos docentes por sostenerla, invisibilizando algunas actitudes de los estudiantes, quienes demandan maneras nuevas de relacionarse, de comprender el conocimiento, de explicar la realidad. Frente a esas posiciones docentes verticales, es perentorio anteponer la reflexión y la resignificación de aquellos referentes conceptuales, que dificultan la comprensión de las relaciones escolares desde una perspectiva de cambio permanente, y por qué no, de caos. 
De ahí que el considerar a la escuela como un lugar de construcción intercultural permanente, influida por múltiples relaciones, pero, a la vez, el lugar donde se mediatizan éstas y se construyen nuevas, se torna necesario. El reconocer que las formas de apropiación del conocimiento y las historias de vida de los sujetos se condicionan mutuamente, es una tarea urgente de resolver, en el sentido de que los individuos construyen sus representaciones mientras viven y satisfacen sus diferentes necesidades biológicas y psicológicas en escenarios sociales que establecen no sólo los problemas que se consideran importantes, sino también los recursos y las estrategias sociales e individuales que merecen la pena.

Se requiere de una nueva escuela para una sociedad multicontextual y multicultural, en la que se fomente el respeto, la tolerancia y la libertad de pensamiento y se cree un principio que facilite, como dicen López y Lleras (2002):

(...) construir la cultura de la diversidad frente a la cultura del handicap (...). La diversidad hace referencia a la identificación de la persona por la persona, por la que cada cual es como es y no como nos gustaría que fuera (identidad). Este reconocimiento es, precisamente, lo que configura la dignidad humana. La diferencia es la valoración de la diversidad y es, precisamente, en esta valoración donde hay varias manifestaciones, ya sean de rechazo (antipatía, xenofobia, racismo, intolerancia...) como de valor (p. 26).

En una sociedad multicontextual y multicultural, la educación debe responder no sólo para compartir nuevos modelos de sociabilidad, sino, además, para aspirar a que se utilicen nuevos estilos cognitivos, basados en la relatividad de los puntos de vista. La educación orientada a la interculturalidad y a la mundialidad no puede olvidarse de una ética de la responsabilidad individual y colectiva. La naturaleza es muy diversa y no existe característica más genuina, en el ser humano, que esa diversidad (López y Lleras, 2002).

Si asumimos que la escuela del siglo XXI debe estar enmarcada dentro de un modelo educativo de equidad y de inclusión, entonces, se puede decir que en ella nadie será excluido y todos tendrán un lugar que ocupar. Un modelo educativo con el que se procure la igualdad de oportunidades y de trato para toda la niñez, de tal forma que las diferencias étnicas sociales, económicas, de género, de discapacidad, de salud, etc. no se traduzcan en discriminaciones o defectos. 
La interculturalidad es la dimensión de la vida humana en sociedad, que tiene que ver con los ámbitos social, político y económico, es un desafío vital, una actitud de apertura que libera al ser humano de los prejuicios, de las certezas absolutas; pero, sobre todo, que nos conduce a romper con la intolerancia. La práctica educativa es considerada, desde la pedagogía intercultural, en relación con las diferencias culturales de individuos y grupos, como foco de reflexión y de indagación pedagógica; propone dar respuesta a las sociedades democráticas que respetan el pluralismo cultural.

Dentro de la pedagogía intercultural se define a la escuela intercultural, como aquella que asume los objetivos propuestos y responde al reto de educar a diferentes grupos, haciendo la escolaridad igual y equiparable para todos, atendiendo a las diferencias existentes entre los alumnos. Se busca potenciar una escuela no sólo de ganadores o de perdedores, sino una escuela que tenga como propósito el aprender la capacidad de aprender; esto implica un cambio de formas de pensar e interactuar de todos, admitir que los estudiantes aprenden de diferentes formas y que sus capacidades no son algo estático, sin olvidar las limitaciones personales. "De ahí que las estrategias y actividades deben estar en consonancia con el análisis y necesidades y previsión de objetivos propuestos en la planificación de la enseñanza, con orientaciones que promuevan contrarrestar el racismo, la discrimación; fomentando el aprendizaje cooperativo" (Aguado, 2003, p. 122).

El continuar considerando la escuela como un escenario donde se construye y se transmite el conocimiento, al margen del entramado cultural que le da sentido al individuo, no es posible, ni válido. Es urgente que los docentes valoren, a la hora de compartir los espacios escolares con sus estudiantes, su cultura experiencial; según Pérez Gómez (1999a), esto tiene que ver con "la peculiar configuración de significados y comportamientos que los alumnos y las alumnas de forma particular han elaborado, inducidos por su contexto, en su vida previa y paralela a la escuela, mediante los intercambios espontáneos con el medio familiar y social que ha rodeado su existencia" (p. 199).

Reconocer esta cultura experiencial como el sustrato, mediante el cual los individuos estructuran sus formas de relacionarse, de comprender los fenómenos, de fabricar la arquitectura cognitiva con la que se apropia del mundo e interviene en él, posibilitaría, una 
forma alternativa de mediación docente, por medio de la cual, los hábitos, las conductas, las formas de conocer y de expresar, de amar y de odiar, de los estudiantes (aunque contradictorias y siempre en construcción) cobrarían validez y significado. Tal vez, se desdibujen las imágenes de docentes perplejos, desprovistos de herramientas explicativas, agobiados e indefensos ante la cultura de los estudiantes y, por el contrario, se erijan otras: retadoras, insatisfechas, desafiantes y comprensivas de las realidades escolares.

Los retos son variados y múltiples, de ahí que las respuestas también lo sean. Uno de los caminos por explorar en esta incierta ruta, es la adopción de paradigmas mucho más integrales de explicación del fenómeno educativo, se hace necesario, moverse por sendas menos aisladas, en las que los estudiantes sean percibidos como totalidades. Posiciones de corte holista abogan por la necesidad de reconocer que ya no es posible educar seres humanos, para una sociedad sustentable en el siglo XXI, con un paradigma científico industrial del siglo XVII.

El mundo de la ciencia es un elemento necesario, pero no suficiente para construir una nueva cultura y una nueva conciencia de naturaleza holista. En este sentido, Gallegos (2000) considera que ya no podemos seguir reduciendo la educación a un entrenamiento de la racionalidad instrumental, para que actúe dentro de la rutina laboral de procesos mecánicos, tal como la sociedad científico-industrial lo necesitó en su tiempo. Hoy, los sistemas educativos, desde la educación preescolar hasta la universitaria (incluso los posgrados), deberán transformarse, radicalmente, para convertirse en un puente que conduzca a los seres humanos a una formación integral, a una conciencia de interdependencia, cooperación y paz global, conceptos, procedimientos y actitudes nuevas en los modelos de formación docente.

\section{Conciliar la teoría y la práctica: un reto en la formación de docentes}

Es común escuchar entre los involucrados en el quehacer educativo, la afirmación de que existe un enorme vacío entre la teoría, que es el resultado, del fruto de la investigación, especialmente circunscrita al ámbito universitario y la práctica, atribuida a quienes les corresponde pasar largas jornadas de trabajo en los centros de enseñanza. Entre ambos grupos 
existen posiciones discrepantes, que contribuyen poco al desarrollo educativo; sin embargo, teoría y práctica son dos conceptos que se desarrollan simultáneamente y constituyen sucesos de un mismo acontecimiento. En resumen,"(...) la teoría no son sólo palabras ni la práctica es mera conducta muda. Desde este punto de vista, no puede haber ninguna distancia entre la teoría y la práctica, sino solo mayores o menores grados de desajuste, elisión e ilusión en la relación entre ellas" (Carr, 1999, p. 34).

La importancia de acercar ambas posiciones reside en la necesidad de enriquecer y de mejorar la actividad docente en sentido amplio. Para ello, se requiere atribuir posturas docentes de reflexión continua de su quehacer; es necesario visualizar una práctica pedagógica que se nutra de las propias experiencias docentes, en las que el conocimiento se valore como un proceso de construcción colectiva y no como el resultado de una imposición. De ahí que la relación entre teoría y práctica revista, sobre todo, un matiz político y transformador y, por tanto, propositivo de cambios en la educación, en particular, y en la sociedad, en general. En la tarea de acercar la teoría a la práctica, la investigación juega un papel decisivo, por lo que la adopción de esquemas mentales, entre el profesorado, que potencien esta postura es urgente.

Un aspecto que en el contexto de la educación nacional es fundamental de abordar, y que guarda estrecha relación con las nociones de teoría y práctica, reside en las características actuales del modelo de formación docente, el cual, lejos de fomentar actitudes de reflexión y transformación de la práctica pedagógica, tiende a reproducir, ingenuamente, las tendencias de sus formadores, dentro de un enfoque que se caracteriza por la repetición de tareas técnicas y estructuras rígidas de aprendizaje. Al respecto, Rodríguez (1995), menciona cuatro tendencias en la formación de docentes que permiten acercarse con claridad al problema; sin embargo, la denominada concepción tradicional oficio, por las características que expresa, es la que permite establecer los paralelismos más cercanos con la realidad nacional.

La concepción tradicional oficio recibe su nombre por la semejanza que tiene con la manera de aprendizaje de los oficios artesanales, propios del período preindustrial, cuando el maestro invitaba a los aprendices a observarlo durante largos lapsos, mientras éstos, mediante la imitación lograban dominar el oficio, gracias a la reproducción del modelo. Si llevamos la analogía al salón de clases, se reconoce que, tradicionalmente, la formación del docente se ha 
llevado a cabo poniendo en contacto al estudiante con la realidad docente para que la observe y la imite. Tras un período de observación se enfrentaba al aprendiz con la realidad escolar, de forma que, imitando el modelo, y por un proceso de ensayo y error, alcanzara la maestría o el dominio de las técnicas apropiadas para la enseñanza.

Los aspectos criticados de esta tendencia se refuerzan cuando, en la formación de los docentes, se separan la teoría y la práctica, cuando el currículum profesional aparece segmentado y se lo considera de poca entidad académica; y cuando no se dan las condiciones que permiten que en las aulas, en las que realizan las prácticas de enseñanza los aspirantes a maestros, estén atendidas por profesionales competentes. Otro aspecto criticado, dentro de esta concepción, es la consideración que dentro de las habilidades necesarias para ejercer la docencia, la más significativa es el dominio de los contenidos que se vayan a enseñar. Al respecto, Rodríguez (1995, p. 21) señala que “lo importante para enseñar es dominar los contenidos que hay que transmitir y que con eso es suficiente, 0 , al menos, eso es lo fundamental. Bastaría añadir un leve barniz de materias psicopedagógicas y algunas prácticas. Después, el oficio se aprende ejercitándolo" (p. 21).

Esta tendencia sigue vigente, en buena medida, y, de una forma más o menos pura e implícita, constituye una concepción previa de las personas que tienen inquietud de estudiar educación, para los cuales la tarea más importante, en la formación docente, la constituye la práctica. Ésta se entiende como la oportunidad de observar al maestro para aprender más de sus aciertos, que de sus errores, posición que encuentra su referente conceptual en el paradigma positivista del aprendizaje, y, como se señala ampliamente: desdibuja la relación vinculante entre la teoría y la práctica docente. Superar la yuxtaposición existente hasta ahora, haciendo que, dentro de lo posible, la teoría y la práctica se solapen en el tiempo, requiere la integración de los cursos pedagógicos, con las llamadas prácticas docentes, con el objeto de proporcionar a los futuros docentes la oportunidad de aplicar, simultáneamente, el conocimiento y las destrezas en el marco del aula real.

En la práctica, este modelo de formación de docentes, en el plano de las universidades públicas, como, agudamente, señala Tünnermann (2005): 
Se caracteriza por estructuras académicas estrictas, dominadas por una visión unidisciplinar, con currículos rígidos y exclusivamente profesionalizantes, sistemas de bloques de asignaturas por semestre y con procesos de enseñanza-aprendizaje que ponen todo el énfasis en la enseñanza, centrados en los profesores, con predominio de las clases expositivas y las conferencias magistrales, que conducen a los estudiantes a adoptar una actitud pasiva en el aula y los transforma en simples receptores y repetidores de los conocimientos expuestos en ella" (p. 25).

Posiblemente, uno de los aspectos con el que la mayoría de los académicos vinculados con los procesos de diseños curriculares de planes de estudio, cualquiera que sea su orientación, estarían de acuerdo, es en la cantidad de requisitos y correquisitos que distinguen las estructuras de los planes y sobre los cuales, la posibilidad de favorecer la participación flexible de los estudiantes y el cambio de viraje, cuando se hace necesario, se torna limitada y asfixiante. Lo anterior se une al tiempo asignado para la finalización de los planes de estudio, especialmente, en las carreras de formación inicial. De ahí la necesidad de incorporar, en el diseño curricular de los programas de formación docente, estructuras más flexibles, innovadoras y que respondan a los nuevos requerimientos sociales, especialmente aquellos que provienen de los sectores más vulnerables.

Es perentorio romper con las barreras academicistas contenidas en la visión curricular mencionada y, por el contrario, impulsar proyectos que aspiren a la formación integral de educadores con visión de futuro; para ello, se debe enfatizar en el desarrollo de capacidades o competencias instrumentales e investigativas, más que en los contenidos disciplinarios per se y criterios técnicos instrumentales, sin perder de vista que lo fundamental no es la formación integral, recargada de competencias laborales, estrictamente orientadas al desarrollo del mundo del trabajo, sino de otras centradas en el desarrollo de actitudes y valores humanísticos dignificantes de la persona.

La falta de una verdadera integración de la teoría y la práctica en la formación de profesores, sobre todo, inicial, a pesar de la preparación para la enseñanza reflexiva, que se contempla, ampliamente, en los programas de los cursos propios de los centros de formación, exige una continua interacción. La investigación reciente recogida por el paradigma crítico, ha puesto de manifiesto lo inadecuado de una formación docente entendida en fases separadas, pues se debe pensar, más bien, en un continuo y progresivo ir y venir reflexión-acción-reflexión- 
acción que articule la teoría y la práctica en un proceso unitario de funcionalidad recíproca entre ambas. No cabe duda de que la necesidad de revisar, de manera cuidadosa, las características de los modelos de formación docente debe constituir un reto vigente de la educación, especialmente de la universitaria.

\section{Un punto de llegada, un nuevo punto de partida}

La tarea de asumir el reto de compartir, intencionadamente, los escenarios escolares, con las personas, que allí construyen sus perspectivas sobre el funcionamiento del mundo no es fácil y, mucho menos, cuando ésta se toma con la convicción de revelar un estado de las cosas cargado de marginación, inequidad y polarización. Se debe reconocer que el ser docente, maestro o mediador, exige aceptar que los otros, llegan a los espacios de encuentro escolares cargados de significados, fruto de sus experiencias personales y colectivas, y que éstas influyen, decisivamente, en sus formas de aprender. Y más aún, cuando se tiene la certeza de que la naturaleza del mundo es cambiante, dinámica e interdependiente, y obliga a la revisión permanente de los esquemas personales de ver el mundo físico y social.

Por ello, el reconocimiento de la influencia recíproca entre el devenir histórico social inmediato, para muchos, el posmodernismo como cambio de época, con sus luces y sombras, y los sistemas educativos y sus diversas prácticas pedagógicas, constituye un espacio de análisis irrenunciable de todo docente. Ello implica el ejercicio permanente de la reflexión crítica sobre la realidad, la cual se inicia con la reflexión particular de sus experiencias como docente, ciudadano y persona; transcurre con la valoración de otros juicios colectivos y aterriza con la pertinencia de desnudar las exigencias que se le demandan a la educación, a la luz de la necesidad de conformar sociedades más justas e inclusivas.

Lo anterior conduce a la valoración de los escenarios educativos desde ópticas diferentes: en primer lugar, como espacio intercultural, como arena simbólica, en palabras de Giroux (2001), donde todos los actores tienen una historia que contar y la cual marca los límites de las nuevas experiencias por compartir. Los docentes no pueden negar la existencia e importancia 
para los nuevos aprendizajes de esta cultura experiencial, que demanda nuevas prácticas pedagógicas, menos impositivas y lineales, y más democráticas, equitativas y contextuales.

Ello supone, también, la revisión de los modelos de formación de docentes que dominan el escenario actual de las universidades públicas y privadas, incluyendo la asimetría que entre unas y otras prevalece, y que se inclina, cualitativamente, de manera positiva, a favor de las primeras, en especial por el aporte de la investigación y la extensión para el fortalecimiento de la docencia. No obstante, tanto en unas como en otras, es urgente revisar, con mucho cuidado, los paradigmas epistemológicos que orientan sus prácticas de formación, y, sobre todo, en la necesidad de empatar la teoría con la práctica pedagógica, y reconocer que la primera exige un acercamiento profundo de la realidad; en este caso, de lo educativo y lo pedagógico, para nutrir la segunda, la cual ofrece el espacio para que suceda la primera, es decir: no hay teoría sin práctica, ni práctica que no envuelva una concepción teórica. Es imperativo tener claro que algunas prácticas corresponden a visiones teóricas del mundo y de la educación poco transformadoras, tanto de prácticas pedagógicas con pertinencia cultural, como de aquellas situaciones que inciden, directamente, en la construcción de sociedades más justas.

Los retos no son pocos ni fáciles de enfrentar; sin embargo, la trascendente tarea de educar obliga a tomar caminos nuevos y superar, de manera permanente, los obstáculos existentes. Las reflexiones aquí presentadas, lejos de ofrecer todas las respuestas, tienen el propósito de generar nuevas interrogantes, que permitan iniciar nuevos puntos de partida.

\section{Referencias}

Aguado, T. ( 2003). Pedagogía Interercultural. Madrid: Editorial McGraw Hill.

Carr, W. (1999). Una teoría para la educación. Madrid: Editorial Morata.

Gallegos, R. (2000). La educación holista. México: Editorial Pax.

Giroux, H. (2001). Cultura, política y práctica educativa. Barcelona: Editorial Graó. (Biblioteca de aula). 
Hargreves, A. (2003). Profesorado, cultura y postmodernidad. Madrid: Editorial Morata.

López F. y Lleras J. (2002). Planteamientos de la pedagogía crítica: comunicar y transformar. Barcelona: Editorial Graó.

Pérez Gómez, Á. (1999a). Comprender y transformar la enseñanza. Madrid: Editorial Morata.

Pozo Municio, I. (2001). Aprendices y maestros. Madrid: Editorial Alianza.

Rodríguez, A. (1995). Paradigma interdisciplinario en la formación docente. Madrid: Editorial Narcea.

Tünnermann C. (2005). La Globalización: Desafíos para la educación superior. Programa Internacional de Gestión de la Calidad y del Cambio en la Educación Superior (UNICAMBIO XXI). Módulo 5. GTZ-CSUCA-DAAD. León, Nicaragua. 